\title{
Laboratórios on-line: Espaços do ensino remoto e possíveis contribuições para formação humana integral na educação básica
}

Online laboratories: Spaces of remote education and possible contributions to integral human training in basic education

Laboratorios en línea: Espacios de educación a distancia y posibles aportes a la formación humana integral en la educación básica

Fernanda Alves Costa

ORCID: https://orcid.org/0000-0002-0889-725X Instituto Federal de Educação, Ciência e Tecnologia do Tocantins, Brasil E-mail: fernandaalves@ifto.edu.br

Jair José Maldaner

ORCID: https://orcid.org/0000-0002-8288-6583 Instituto Federal de Educação, Ciência e Tecnologia do Tocantins, Brasil E-mail:jair@ifto.edu.br

Marcelo Rythowem

ORCID: https://orcid.org/0000-0001-5819-3800 Instituto Federal de Educação, Ciência e Tecnologia do Tocantins, Brasil E-mail: marcelo@ifto.edu.br

Rivadavia Porto Cavalcante ORCID: https://orcid.org/0000-0002-6568-7910 Instituto Federal de Educação, Ciência e Tecnologia do Tocantins, Brasil E-mail: riva@ifto.edu.br

Rosa Maria Machado de Sena ORCID: https://orcid.org/0000-0002-3616-650X Instituto Federal de Educação, Ciência e Tecnologia do Tocantins, Brasil

E-mail: rosa.sena@ifto.edu.br

Valcí Ferreira Victor

ORCID: https://orcid.org/0000-0003-2935-5895 Instituto Federal de Educação, Ciência e Tecnologia do Tocantins, Brasil E-mail: victor@ifto.edu.br

Cláudio de Castro Monteiro ORCID: https://orcid.org/0000-0002-5171-4877 Instituto Federal de Educação, Ciência e Tecnologia do Tocantins, Brasil E-mail: ccm@ifto.edu.br

Wallysonn Alves de Souza ORCID: https://orcid.org/0000-0002-2966-8130 Instituto Federal de Educação, Ciência e Tecnologia do Tocantins, Brasil E-mail: wallysonn.souza@ifto.edu.br

\begin{abstract}
Resumo
O uso das Tecnologias de Informação e Comunicação (TICs) tornou-se essencial nas atividades das instituições de educação por meio do ensino remoto com a suspensão das aulas presenciais por causa da pandemia da Covid-19. Devido às dificuldades de acesso aos laboratórios tradicionais para realização de práticas pelos estudantes da educação básica, tornou-se essencial a utilização de laboratórios remotos e virtuais para realização de práticas, que podem ser acessados via internet independente da localização geográfica dos estudantes, uma série destes têm sido desenvolvidos por algumas instituições, destaca-se a Universidade Federal de Santa Catarina (UFSC) através do grupo de pesquisa do Laboratório de Experimentação Remota (RexLab). Esse novo contexto passou a exigir pesquisas sobre projetos voltados para integração das TICs na educação básica sob a perspectiva da formação humana integral. Este artigo tem como objetivo de relatar sobre experiências exitosas dos projetos voltados para uso de tecnologias educacionais que utilizam os laboratórios remotos e virtuais voltado para o ensino médio no intuito de aplicação de práticas de disciplinas das ciências aplicadas, desenvolvido pelo grupo de pesquisa do RExLab da UFSC com apoio do Conselho Nacional de Desenvolvimento Científico e Tecnológico (CNPQ).

Palavras-chave: Tecnologias de informação e comunicação; Educação básica; Formação humana integral; Ensino remoto; Laboratórios on-line.
\end{abstract}




\begin{abstract}
The use of Information and Communication Technologies (ICTs) has become essential in the activities of education institutions through remote teaching with the suspension of face-to-face classes due to the Covid-19 pandemic. Due to difficulties in accessing traditional laboratories for carrying out practices by basic education students, it has become essential to use remote and virtual laboratories to carry out practices, which can be accessed via the internet regardless of the geographical location of the students, a series of these have been developed by some institutions, the Federal University of Santa Catarina (UFSC) stands out through the research group of the Remote Experimentation Laboratory (RexLab). This new context began to demand research on projects aimed at the integration of ICTs in basic education from the perspective of integral human formation. This article aims to report on successful experiences of projects aimed at the use of educational technologies that use remote and virtual laboratories aimed at high school in order to apply practices of applied science disciplines, developed by the RExLab research group at UFSC with support from the National Council for Scientific and Technological Development (CNPQ).

Keywords: Information and communication technologies; Basic education; Integral human training; Remote teaching; Online labs.

\section{Resumen}

El uso de las Tecnologías de la Información y la Comunicación (TIC) se ha vuelto imprescindible en las actividades de las instituciones educativas a través de la enseñanza a distancia con la suspensión de las clases presenciales por la pandemia del Covid-19. Debido a las dificultades de acceso a los laboratorios tradicionales para la realización de prácticas por parte de los estudiantes de educación básica, se ha vuelto imprescindible el uso de laboratorios remotos y virtuales para la realización de prácticas, a los cuales se puede acceder vía internet sin importar la ubicación geográfica de los estudiantes, un serie de estos han sido desarrollados por algunas instituciones, se destaca la Universidad Federal de Santa Catarina (UFSC) a través del grupo de investigación del Laboratorio de Experimentación Remota (RexLab). Este nuevo contexto comenzó a demandar la investigación de proyectos dirigidos a la integración de las TIC en la educación básica desde la perspectiva de la formación humana integral. Este artículo tiene como objetivo relatar experiencias exitosas de proyectos dirigidos al uso de tecnologías educativas que utilizan laboratorios remotos y virtuales destinados a la enseñanza media para aplicar prácticas de disciplinas de ciencias aplicadas, desarrollados por el grupo de investigación RExLab de la UFSC con apoyo de la Universidad Nacional. Consejo de Desarrollo Científico y Tecnológico (CNPQ).

Palabras clave: Tecnologías de la Información y la Comunicación; Educación básica; Formación humana integral; Enseñanza a distancia; Laboratorios en línea.
\end{abstract}

\title{
1. Introdução
}

O advento da suspensão das atividades presenciais devido a pandemia da covid-19, provocou um novo cenário para educação básica brasileira, a continuação das atividades por meio do ensino remoto. Assim, professores e estudantes lidam com o desafio do ensino-aprendizagem via internet. (Rocha \& Lima, 2021; Firmino et al., 2022)

Diante desta nova realidade os docentes da educação básica à pós-graduação scricto sensu vão em busca de capacitações e soluções para lidar com o uso das tecnologias digitais através do uso de metodologias ativas que colaboram para maior participação e motivação dos estudantes. A preocupação com a qualidade do ensino e com a continuação das atividades, contribuiu para que diversas instituições buscassem soluções sobre a disponibilização de internet aos estudantes para acesso aos conteúdos e aulas on-line. (Rocha \& Lima, 2021; Firmino et al., 2022)

Anteriormente, o uso das tecnologias digitais para fins educacionais relacionava-se apenas com educação à distância (EAD), pois esta modalidade de ensino necessita de ferramentas que viabilizem o fluxo de informação e comunicação no seu modo de operação, onde professores e estudantes, no desenvolvimento de suas atividades de ensino-aprendizagem estão geralmente em localização e temporalidade diversificado. Sendo assim, no ensino remoto, aplica-se o universo presencial, o ensino on-line, não se limitando ao escopo do EAD. (Wenczenovicz, 2020; Rocha \& Lima, 2021)

O maior desafio para educação básica, especificamente no ensino médio tanto das redes públicas como nas privadas, é lidar com o ensino das ciências aplicadas (química, física, biologia e etc.) e na educação profissional tecnológica somam-se os saberes técnicos com as aulas práticas desenvolvidas em laboratórios, outros espaços formais e informais dentro das instituições de ensino. (Mota, 2019; Digital, 2018; Frigotto et al., 2005).

Conforme (Mota, 2019; Digital, 2018; Frigotto et al., 2005), o acesso aos laboratórios tradicionais é escasso para os 
estudantes do sistema brasileiro de ensino, distanciando-os da formação humana integral, principalmente da classe trabalhadora.

A realização das atividades práticas é imprescindível para que os estudantes contextualizem a teoria, proporcionando o desenvolvimento de habilidades que serão necessárias na formação dos cidadãos nas diversas dimensões voltado para o mundo trabalho. No entanto, alguns aspectos dificultam a disponibilização dessas práticas em laboratórios presenciais como por exemplo, a falta de orçamento para adquirir equipamentos e materiais de consumo, estrutura física inadequada, ausência de atendimento especializado e suporte técnico especializado aos professores e estudantes e a carência de projetos de ensino interdisciplinares. (Mota, 2019; Digital, 2018; Frigotto et al., 2005; Saviani, 1989)

Partindo desta exposição, este trabalho levanta o seguinte problema: diante da precarização e da dificuldade de acesso aos laboratórios tradicionais para realização de práticas pelos estudantes da educação básica, quais as possibilidades do ensino remoto para acesso aos conhecimentos de práticas laboratoriais voltados para ciências aplicadas para educação básica na perspectiva de uma formação omnilateral?

$\mathrm{O}$ uso de laboratórios on-line (remotos e virtuais) tem a oferecer experiências laboratoriais, independentes da localização geográfica dos estudantes. Uma série destes têm sido desenvolvidos por diversas instituições, como na Universidade Federal de Santa Catarina (UFSC) através do grupo de pesquisa do Laboratório de Experimentação Remota (RexLab).

Atuando desde 1997, fortalecendo o atendimento da necessidade de apropriação social da ciência e da tecnologia, popularizando conhecimentos científicos e tecnológicos, estimulando os jovens a inserirem-se nas carreiras científicotecnológicas e buscando iniciativas que integrem a educação científica ao processo educacional promovendo a melhoria devido à atualização/modernização do ensino em todos os seus níveis, enfatizando ações e atividades que valorizem e estimulem a criatividade, a experimentação e a interdisciplinaridade.

Esta pesquisa justifica-se pela necessidade da divulgação para os docentes e estudantes sobre projetos acessíveis via internet e o uso de laboratórios on-line (remotos e virtuais) voltados para experimentação remota de práticas das ciências aplicadas, como também a importância da formação integral dos cidadãos.

No que tange ao exposto, este trabalho tem como objetivo relatar experiências de integração de tecnologias para a educação básica em uma perspectiva na formação humana integral, vivenciadas pelo grupo de pesquisa do Laboratório de Experimentação Remota (RexLab) da Universidade Federal de Santa Catarina (UFSC), com apoio do Conselho Nacional de Desenvolvimento Científico e Tecnológico (CNPQ), pretende-se conhecer, mais efetivamente, projetos voltados para o uso de tecnologias educacionais.

\section{Metodologia}

A pesquisa é um processo formal e sistemático de desenvolvimento científico, com objetivo de descobrir respostas para problemas por meio de procedimentos científicos, a pesquisa social é o processo que utiliza a metodologia científica para obtenção de novos conhecimentos no campo da realidade social. (Antônio Carlos Gil, 2008; Uwe Flick et al., 2009)

A pesquisa exploratória é necessária para coleta de dados desta pesquisa, assim, este trabalho tem como finalidade esclarecer e discutir conceitos quanto ao uso de tecnologias educacionais, através do uso de laboratórios remotos e virtuais, tendo em vista a formulação de hipóteses para estudos posteriores sobre projetos educacionais desenvolvidos atualmente pelo grupo de pesquisa do Laboratório de Experimentação Remota (RexLab) da Universidade Federal de Santa Catarina (UFSC) a partir da integração de tecnologias inovadoras com perspectiva na formação omnilateral dos estudantes da educação básica. (Lakatos \& Marconi, 2001; Antônio Carlos Gil, 2008)

A abordagem qualitativa é recomendada para interpretar fenômenos realísticos, vivenciais e sociais através da 
interação entre a observação e a formulação do conceito, entre o desenvolvimento teórico e a pesquisa e entre a explicação e a percepção. A pesquisa exploratória é uma metodologia que costuma envolver o levantamento bibliográfico com as experiências com o problema pesquisado através de análise de exemplos que estimulem a compreensão. (Bulmer, 2001; Lakatos \& Marconi, 2001; Gibbs 2008; Antônio Carlos Gil, 2008; Uwe Flick et al., 2009)

Sendo assim, este estudo tem como perspectiva a pesquisa exploratória, de abordagem qualitativa que utiliza procedimentos de revisão bibliográfica. Sendo esta pesquisa bibliográfica voltada aos projetos voltados para uso de tecnologias educacionais sobre a utilização de laboratórios remotos e virtuais para o ensino médio, no intuito de aplicação de práticas de disciplinas das ciências aplicadas, desenvolvido pelo grupo de pesquisa do RExLab da UFSC sob a perspectiva da formação humana integral/omnilateral.

A coleta de dados desta pesquisa são de 3 (três) estudos realizados entre 2017 e 2018, sobre os principais programas/projetos desenvolvidos pelo grupo RExLab: Integração de Tecnologia na Educação Básica (InTecEdu) e a plataforma modular para construção, gestão e disponibilização de laboratórios remotos na educação.

Os dados obtidos pela revisão bibliográfica das publicações científicas dos atores da Educação Profissional e Tecnológica e pesquisadores do Laboratório Experimentação Remota (RExLab)/UFSC, foram submetidos à análise crítica e posteriormente foram feitas discussões dos dados levantados.

\section{Resultados e Discussão}

Nesta seção serão abordados os resultados obtidos nesta pesquisa, relatando os projetos desenvolvidos pelo grupo de pesquisa do Laboratório de Experimentação Remota (RExLab) da Universidade Federal de Santa Catarina (UFSC) com objetivo de integrar tecnologias e atividades didáticas voltadas para educação básica.

Os resultados deste estudo foram obtidos por informações e por publicações de trabalhos científicos que podem ser acessados pelo site desenvolvido pelo grupo de pesquisadores da RexLab (https://rexlab.ufsc.br/), de forma clara e objetiva são encontrados os entendimentos sobre os diversos temas sobre o uso de tecnologias educacionais, a formação de professores, integração de práticas educativas com uso dos laboratórios on-line (remotos e virtuais).

Os projetos relatados nesta pesquisa possuem três fortes pontos, com características exploradas pela equipe do Rexlab/UFSC: a realização de atividades de investigação por meio de experimentação prática via internet, a construção de plano de ensino pelos professores através de uma indagação central voltada para desenvolvimento de atividades práticas voltadas para ciências aplicadas, contendo os aspectos de conteúdo, pedagógicos e tecnológicos, e, por fim, a formação do estudante sob a perspectiva de uma formação integrada, trabalhando suas diversas dimensões através da educação e tecnologia. (Saviani, 1989; Charlot, 2014; Santos et al., 2018)

\subsection{O programa de integração de tecnologia na educação básica}

O programa de Integração de Tecnologia na Educação Básica (InTecEdu) é formado por um conjunto articulado de projetos de pesquisa e extensão que são desenvolvidos em formato de processos e de maneira contínua desde 2008, visando a integração das tecnologias digitais na educação básica e no ensino superior. Sendo sua plataforma de acesso desenvolvida pela equipe do Rexlab/UFSC (https://intecedu.ufsc.br/).

O InTecEdu tem com estratégia a integração de tecnologias nos processos de ensino e aprendizagem, sendo suas ações estruturadas em dois eixos: capacitação de professores e a integração e tecnologias em atividades didáticas desenvolvidas acessados por dispositivos móveis. São financiados pelo CNPQ e também pelo FRIDA, sendo um programa que apoia iniciativas na América Latina e o Caribe que contribuem para a consolidação de uma internet global, aberta, estável e segura, buscando apoiar estratégias alternativas e inovadoras para incrementar o acesso à internet de populações da América 
Latina e do Caribe.

Em um estudo realizado sobre o projeto "Promovendo inclusão digital em escolas públicas de educação básica através da integração de tecnologias inovadoras de baixo custo no ensino de ciências naturais e exatas" realizado pelo RExLab/UFSC, obteve resultados positivos, quanto à integração de tecnologias na educação básica por parte dos professores da rede pública, afirmando que esta prática deve ser incentivada. O projeto capacita os professores quanto às diversas ferramentas na plataforma livre Moodle, demonstrando os caminhos necessários para o desenvolvimento de trabalhos exitosos, possibilitando o aprendizado de diversas ferramentas úteis e a utilização dos laboratórios on-line (virtuais e remotos). (Nardi et al., 2017)

Após o conhecimento adquirido através da capacitação, o professor tem a capacidade de produzir diversos conteúdos para seus cursos, inserindo no Moodle todos os itens necessários para complementar o que é repassado em sala de aula, em seguida, os estudantes utilizam os tablets fornecidos pela equipe do RexLab/UFSC para acessar as sequências didáticas construídas pelos professores. (Nardi et al., 2017)

Ao acessar o portal do InTecEdu através dos tablets, os alunos descobrem vídeos, jogos e atividades correspondentes ao assunto exposto em sala de aula, sentem-se motivados pela oportunidade de participarem de uma aula com uma perspectiva mais dinâmica que adentra o universo tecnológico ao qual estão inseridos na maior parte do tempo. (Silva et al, 2017)

A pesquisa demonstra que 363 professores foram capacitados até o ano de 2017, sendo estes pertencentes ao quadro de seis escolas públicas localizadas no extremo sul de Santa Catarina nos municípios Araranguá e Balneário Arroio do Silva, inicialmente observou-se uma relutância dos participantes no início da capacitação, porque eles acreditavam que aumentariam sua carga de trabalho. Após perceberam que o uso das tecnologias otimizava o tempo de trabalho, já que as atividades poderiam ser corrigidas automaticamente e os materiais poderiam ser facilmente replicados para diversas turmas, houve motivação de outros professores para iniciar a capacitação. (Nardi et al., 2017)

Outro estudo realizado sobre o mesmo projeto teve como objetivo apresentar os resultados da capacitação realizada para os professores da Escola Estadual Indígena Nhu Porã localizada na cidade de Torres/RS, uma instituição de ensino exclusiva para ameríndios e que atendia cerca de 60 alunos do pré-escolar ao sétimo ano do ensino fundamental, com um quadro limitado de professores e de outros profissionais, não tendo laboratório de informática e biblioteca e possuindo somente dois computadores com acesso à internet. Devido à carência de profissionais ocorria o acúmulo de diversas disciplinas pelos professores, a pesquisa demonstrou que a experiência foi vista como positiva pelos professores e os motivando-os a produzirem conteúdos no Moodle na língua tupi-guarani. (Steffens da Rocha et al., 2018)

Foi identificado que os professores precisam de apoio continuado para lidar com as tecnologias voltadas para educação, inclusive no que se refere à orientação quanto às maneiras de fazer a conexão entre o uso das novas tecnologias e os conteúdos a serem ministrados em sala de aula. Importante ressaltar que o Instituto Federal de Santa Catarina (IFSC) e a RExLab/UFSC desenvolvem projetos de extensão na Aldeia Indígena Nhu Porã com a finalidade de diminuir a carência de acesso a recursos de Tecnologias da Informação e Comunicação (TIC) na escola da comunidade. (Steffens da Rocha et al., 2018)

\subsection{A plataforma modular para construção, gestão e disponibilização de laboratórios remotos na educação}

Este projeto surgiu pela necessidade da formação integral dos estudantes, principalmente na área da ciência, tecnologia e de engenharia por meio da qualificação, com a realização de atividades práticas laboratoriais. Tem como característica uma infraestrutura modular, de software e hardware para construção, disponibilização e gestão online de laboratórios remotos, os recursos e os serviços proporcionam o acesso pela internet destes espaços independentemente da localização geográfica do usuário. Sendo assim seus recursos e serviços estão disponíveis para educação básica, educação profissional e tecnológica e ao ensino superior. 
O desenvolvimento destes laboratórios preconiza o atendimento das necessidades específicas de cada experimento ou instituição, habitualmente dependem de uma arquitetura cliente-servidor, permitindo o acesso do equipamento real pela internet, sendo que o servidor de um laboratório pode ser um microcontrolador, um computador ou sistema gerenciamento laboratório remoto RLMS (do inglês Remote Laboratory Management System), assegurando a interação remota do usuário, partindo de um aplicativo autônomo ou como um componente em um ambiente da Web. O usuário observa o laboratório por vídeo ao vivo, definindo os parâmetros de configurações, visualizando os dados e medições em tempo real. (Golinelli et al., 2018)

Em um estudo realizado com objetivo de identificar e analisar a percepção de alunos de uma escola pública Estadual de Santa Catarina, sobre o uso e experimentação em laboratórios remotos com objetivo de integrar ao ensino-aprendizagem na disciplina de física, na percepção dos estudantes, a experimentação remota contribuiu para uma maior motivação do estudo de física, proporcionando o contato com atividades práticas, já que a instituição de ensino não possui laboratório físico. (Heck et al., 2016)

Quanto a proposta de framework (ferramenta que auxilia o programador a trabalhar com determinada linguagem de programação) para integração de tecnologias digitais na educação básica, inspirado na cultura maker, incentiva os estudantes da educação básica, a darem continuidade ao fluxo escolar em suas trajetórias acadêmicas nas áreas de ciência, tecnologia, engenharia e matemática através de capacitação sobre conhecimentos e oportunidades no setor tecnológico e de engenharias.

Em suas atividades promove a atratividade pelas carreiras científico-tecnológicas, no desenvolvimento de atividades pelos professores, visando a integração de tecnologias educacionais em seus planos de aulas para estimular iniciativas e exemplos junto aos estudantes.

\subsection{O uso das tecnologias de informação e comunicação na educação básica sob a perspectiva da formação humana integral}

A continuação das aulas via ensino remoto devido ao contexto pandêmico da covid-19 expôs a complexidade inerente ao contexto da integração tecnológica na educação básica, trazendo uma transformação na dinâmica da construção do ensinoaprendizagem, demonstrando que os métodos tradicionais apresentam dificuldades para conectar os estudantes e envolvê-los de forma completa na realidade e na cultura digital que permeia a sociedade atual. (Nicolete et al., 2016; Rocha \& Lima, 2021; Firmino et al., 2022)

Nesse sentido, ocorre a necessidade de se buscar novas metodologias interdisciplinares de ensino, envolvendo o contexto e a realidade dos estudantes, exigindo das instituições de ensino da educação básica uma maior aproximação, disponibilidade e, consequentemente, maior uso de tecnologias educacionais. Também é preciso integrar, por meio de capacitações, professores e estudantes no processo de ensino e aprendizagem com as tecnologias de comunicação e informação (Tics), inserindo, desta forma, um novo subsídio na formação humana integral. (Nicolete et al., 2016; Firmino et al., 2022)

A interdisciplinaridade no ensino edifica o conhecimento dos estudantes com base na relação do contexto da sua realidade e sua cultura, com a inserção das ferramentas de tecnologia e comunicação no cotidiano escolar ocorre uma transformação na dinâmica da construção do conhecimento, criando uma geração de estudantes que estão imersos em uma nova cadeia tecnológica, na qual o uso de computadores e dispositivos móveis fazem parte da cultura atual e da realidade do cotidiano e podem ser utilizados nas mais diversas áreas do conhecimento. (Nicolete et al., 2016; Paulo Freire; 2019)

Porém, um estudo realizado pela UNESCO (2014), sobre o uso de dispositivos móveis na educação, indicou que projetos educacionais que utilizam as TICs são considerados complementares aos investimentos educacionais já existentes para a educação de qualidade, como infraestruturas, treinamento, livros e conteúdos, não substituindo-os.

A inserção de recursos tecnológicos na educação básica requer mais do que a integração da tecnologia no cotidiano de 
professores e estudantes de forma indiscriminada, é necessário também compreender o processo cíclico da educação em todos os níveis, compreendendo que ações que preparam a classe trabalhadora para o mundo trabalho como também para o ingresso nas instituições de ensino superior que estão diretamente ligadas quanto ao estímulo da formação politécnica. (Saviani, 1989; Frigotto et al, 2005; Martins, 2013; Charlot, 2014; Nicolete et al., 2016)

A integração das tecnologias digitais na educação básica tem contribuído com ações para disciplinas essenciais em todos os níveis, especificamente as disciplinas das ciências aplicadas, como a física, biologia, matemática, química, sendo utilizadas estratégias da experimentação remota, destacando as práticas realizadas em laboratórios remotos, onde são acessados em tempo real via internet, por meio de equipamentos e experimento físicos reais, como também os laboratórios virtuais, modelos constituídos de simulação. (Nicolete et al., 2016; Digital, 2018)

A aplicação de aulas práticas em laboratórios remotos e virtuais pelos estudantes da educação básica tem sua importância na integração dos diversos elementos pedagógicos aos recursos em sala de aula, não ocorrendo a substituição dos laboratórios tradicionais, porém, como complemento, podendo abordar técnicas e práticas que não são compreensíveis mesmo em atividades realizadas em ambientes físicos de experimentação. (Mellos et al., 2018)

Esses espaços mostram-se como uma ferramenta transdisciplinar com o potencial de promover a expansibilidade do ambiente escolar para além dos espaços formais e informais das instituições de ensino, com a possibilidade de acesso em todos os horários e dias da semana, sendo necessário ponderação quanto os níveis de aplicação considerando as individualidades dos professores e as potencialidades de cada tipo de laboratório. Por isso a importância das escolas construírem seus currículos e propostas político-pedagógicas, considerando suas realidades e de forma participativa. (Frigotto et al, 2005; Mellos et al., 2018)

A consolidação de projetos para os estudantes da educação básica, permite uma visão prospectiva, projetando o futuro, ambos contribuindo para situar o indivíduo, suas motivações e o significado de suas ações, dentro das conjunturas de vida, na sucessão das etapas de sua trajetória. (Frigotto et al, 2005)

\section{Conclusão}

O relato das experiências de integração de tecnologias para educação básica abordadas nesta pesquisa, apontam a possibilidade do uso das tecnologias digitais na formação humana integral, tendo como destaque o programa de Integração de Tecnologia na Educação (InTecEdu) desenvolvido desde 2008 pelo grupo de pesquisa do RExLab da UFSC com apoio do Conselho Nacional de Desenvolvimento Científico e Tecnológico (CNPQ).

Demonstrando que este programa, vem desenvolvendo projetos de pesquisa e extensão que integram a tecnologia e atividades didáticas tanto na educação básica como no ensino superior, desenvolvido de forma processual e contínua, possuindo como característica a capacitação de professores e estudantes das instituições públicas de ensino, para desenvolvimento de projetos que estimulam a cultura científica.

A construção e disponibilidade através de plataforma aberta de laboratórios remotos e virtuais voltados para o ensino médio, integram a aplicação de práticas pedagógicas voltadas para disciplinas das ciências aplicadas, como por exemplo, Física, Química, Biologia e Matemática, vem motivando os estudantes a seguirem carreiras científico-tecnológicas.

O ensino remoto para continuação das atividades da educação básica deu um salto quanto ao uso das tecnologias de informação e comunicação nas atividades de ensino, pesquisa e extensão, trazendo impactos significativos e com o desafio de integrar e disponibilizar o acesso às tecnologias educacionais no cotidiano de professores e estudantes.

A pesquisa bibliográfica aqui apresentada evidencia os projetos desenvolvidos com percepção da formação humana integral, omnilateral, politécnica, na integração da ciência, cultura e tecnologia, por busca de novos recursos didáticos para melhoria da educação. Evidenciamos que estas ações são voltadas para todos os níveis educacionais, destacando-se as 
disciplinas das ciências aplicadas com a inserção dos experimentos realizados via internet nos laboratórios remotos e virtuais.

Diante do reconhecimento da integração tecnológica na educação, a possibilidade de sucesso que descrevemos, se dará com o desenvolvimento de projetos e ações na educação básica voltada para os professores, estudantes e os diversos profissionais das instituições da educação pública, como também o desenvolvimento de trabalhos interdisciplinares em conjunto com as Universidades Federais e os Institutos Federais de Educação, Ciência e Tecnologia.

Por fim, sugere-se que para o sucesso de futuros trabalhos de integrações com as TICs na educação básica brasileira depende do investimento e disponibilidade de infraestrutura tecnológica nas escolas, sendo reconhecidas as carências de infraestrutura, orçamento e de projetos quanto aos laboratórios voltados para o ensino, como também, a importância da motivação e capacitação dos professores e estudantes.

\section{Agradecimentos}

Ao grupo de pesquisadores da RexLab da Universidade Federal de Santa Catarina pelo apoio prestado durante o desenvolvimento das etapas deste trabalho, como também pela oportunidade de participação nos projetos de extensão desenvolvidos no ano de 2021.

\section{Referências}

Gil, A. C. (2008). Métodos e técnicas de pesquisa social. Atlas.

Bulmer, M. (2001). Sociological research methods: an introduction. Macmillan.

Charlot, B. (2014). Trabalho e educação: abordagens antropológica e sócio-histórica da relação com o saber às práticas. Ed. Cortez.

Digital, P. E. (2018). O laboratório de química nas aulas de ensino médio. Plataforma Espaço Digital. https://editorarealize.com.br/artigo/visualizar/52367

Dermeval Saviani. (1989). Sobre a concepção de politecnia [apresentado do "Seminário Choque Teórico" da Saúde, 2, 3 e 4 de dezembro de 1987]. Rio De Janeiro Politécnico Da Saúde Joaquim Venâncio

Firmino, N. C. S., Firmino, D. F., Barbano, E. P., Mendonça, G. L. F., \& Leite, L. R. (2022). O ensino remoto emergencial: ações e adaptações de estudantes cearenses. Research, Society and Development, 11(1), e18011125028. https://doi.org/10.33448/rsd-v11i1.25028

Gaudêncio Frigotto, Ciavatta, M., Ramos, M., Eliza Bartolozzi Ferreira, Regina, S., \& CorrêaV. (2005). Ensino médio integrado : concepção e contradições. Cortez Editora.

Gibbs, G. (2008). Análise de Dados Qualitativos. Artmed.

Golinelli, M. H. de M., Silva, J. B. da, \& Yevseyeva, O. (2018). Arquitetura de dispositivos inteligentes para laboratórios remotos: uma revisão sistemática da literatura. RETEC - Revista de Tecnologias, 11(2). https://www.fatecourinhos.edu.br/retec/index.php/retec/article/view/306

Heck, C., Coelho, K. dos S., Simão, J. P. S., Silva, I. N. da, Silva, J. B. da, \& Bilessimo, S. M. S. (2016). Experienência de Integração da Experimentação Remota No Ensino De Física Do Ensino Médio: Percepção dos Alunos. RENOTE, 14(2). https://doi.org/10.22456/1679-1916.70662

Lakatos, E. M. \& Marconi, M. A. (2003). Fundamentos de metodologia científica. Ed. Atlas.

Martins, L. M. (2013). Os fundamentos psicológicos da pedagogia histórico-crítica e os fundamentos pedagógicos da psicologia histórico-cultural. Germinal: Marxismo E Educação Em Debate, 5(2), 130. https://doi.org/10.9771/gmed.v5i2.9705

Mellos, L., Bento Da Silva, J., Bosco, J., Alves, M., \& Ferenhof, H. (2018). Um olhar sob a interatividade em laboratórios online. http://tecedu.pro.br/wpcontent/uploads/2018/06/Art9-vol.24-Edi\%C3\%A7\%C3\%A3o-Tem\%C3\%A1tica-VII-Junho-2018.pdf

Mota, M. D. (2019). Laboratórios de Ciências/Biologia nas escolas públicas do Estado do Ceará (1997-2017): realizações e desafios. https://repositorio.ufc.br/bitstream/riufc/45994/1/2019_tese_mdamota.pdf

Nardi, I., Karmel, C., Nardi Da Silva, K. C., Schmidt, K., \& Bento Da Silva -Juarez, J. (2017). Inclusão digital em escolas públicas através de tecnologias inovadoras de baixo custo no ensino de disciplinas. Revista Renote https://seer.ufrgs.br/renote/article/download/79264/46158

Nicolete, P. C., Bilessimo, S. M. S., Freire, P. de S., Silva, J. B. da, \& Cristiano, M. A. da S. (2016). Integração Tecnológica na educação básica pública brasileira: uma análise sobre a evolução temporal e a interdisciplinaridade do tema. Revista Ibero-Americana de Estudos Em Educação, 11(1982-5587), 2064 2086. https://doi.org/10.21723/riaee.v11.n4.8151

Paulo Freire. (2019). Pedagogia do oprimido. Paz E Terra. 
Research, Society and Development, v. 11, n. 2, e43511225904, 2022

(CC BY 4.0) | ISSN 2525-3409 | DOI: http://dx.doi.org/10.33448/rsd-v11i2.25904

Rocha, E. M., \& Lima, J. M. da S. (2021). Impactos e desafios do ensino on-line decorrentes da pandemia COVID-19. Revista Ibero-Americana De Estudos Em Educação, 16(2), 377-390. https://doi.org/10.21723/riaee.v16i2.14526

Santos, A. C. dos, Sousa, B. J. de, Canto, J. Z. do, \& Silva, J. B. da. (2018). Ensino de ciências baseado em investigação: uma proposta didática inovadora para o uso de laboratórios on-line em avea. Revista Univap, 24(44), 54-68. https://doi.org/10.18066/revistaunivap.v24i44.1874

Steffens Da Rocha, J., Meister, S., \& Bilessimo, S. (2018). Uso de tecnologias digitais entre professores de uma escola multisseriada indígena: um relato de experiência sobre capacitação docente. Revista Tecnologias na Educação 10(28). https://tecedu.pro.br/wp-content/uploads/2019/01/Re15-Ano-10-vol28Dezembro-2018.pdf

UNESCO. (2014). Diretrizes de políticas para a aprendizagem móvel. http://www.bibl.ita.br/UNESCO-Diretrizes.pdf

Uwe F., Joice E. C., \& Sônia E. C. (2009). Uma introdução à pesquisa qualitativa. Porto Alegre Bookman.

Wenczenovicz, T. J. (2020). Ensino a distância, dificuldades presencias: perspectivas em tempos de COVID-19. Revista Ibero-Americana de Estudos Em Educação, 15(4), 1750-1768. https://doi.org/10.21723/riaee.v15i4.13761 